\author{
MITSUBISHI ELECTRIC RESEARCH LABORATORIES \\ http://www.merl.com
}

\title{
Automatic Image Segmentation by Wave Propagation
}

\author{
Porikli, F.
}

TR2004-010 March 2004

\begin{abstract}
We develop a level set based region growing method for automatic partitioning of color images into segments. Previous attempts at image segmentation either suffer fromrequiring a priori information to initialize regions, being computationally complex, or fail to establish the color consistency and spatial connectivity at the same time. Here, we represent the segmentation problem as monotonic wave propagation in an absorbing medium with varying front speeds. We iteratively emit waves from the selected base points. At a base point, the local variance of the data reaches a minimum, which indicates the base point is a suitable representative of its local neighborhood. We determine local variance by applying a hierarchical gradient operator. The speed of the wave is determined by the color similarity of the point on the front to the current coverage of the wave, and by edge information. Thus, the wave advances in an anisotropic spatial-color space. The absorbing function acts as a stopping criterion of the wave front. We take advantage of fast marching methods to solve the Eikonal equation for finding the travel times of the waves. Our method is superior to the linkage-based region growing techniques since it prevents leakage and imposes compactness on the region without over-smoothing its boundary. Furthermore, we can deal with sharp corners and changes in topology. The automatic segmentation method is Eulerian, thus it is computationally efficient. We compare our results with a non-Eulerian approach that evaluates the arrival times of multiple waves as well. Our experiments illustrate the robustness, accuracy, and effectiveness of the proposed method.
\end{abstract}

This work may not be copied or reproduced in whole or in part for any commercial purpose. Permission to copy in whole or in part without payment of fee is granted for nonprofit educational and research purposes provided that all such whole or partial copies include the following: a notice that such copying is by permission of Mitsubishi Electric Research Laboratories, Inc.; an acknowledgment of the authors and individual contributions to the work; and all applicable portions of the copyright notice. Copying, reproduction, or republishing for any other purpose shall require a license with payment of fee to Mitsubishi Electric Research Laboratories, Inc. All rights reserved.

Copyright (C) Mitsubishi Electric Research Laboratories, Inc., 2004

201 Broadway, Cambridge, Massachusetts 02139 



\section{Publication History:}

1. First printing, TR-2004-010, March 2004 


\title{
Automatic Image Segmentation by Wave Propagation
}

\author{
Fatih Porikli* \\ Mitsubishi Electric Research Laboratories, Cambridge, USA
}

\begin{abstract}
We develop a level set based region growing method for automatic partitioning of color images into segments. Previous attempts at image segmentation either suffer from requiring a priori information to initialize regions, being computationally complex, or fail to establish the color consistency and spatial connectivity at the same time. Here, we represent the segmentation problem as monotonic wave propagation in an absorbing medium with varying front speeds. We iteratively emit waves from the selected base points. At a base point, the local variance of the data reaches a minimum, which indicates the base point is a suitable representative of its local neighborhood. We determine local variance by applying a hierarchical gradient operator. The speed of the wave is determined by the color similarity of the point on the front to the current coverage of the wave, and by edge information. Thus, the wave advances in an anisotropic spatial-color space. The absorbing function acts as a stopping criterion of the wave front. We take advantage of fast marching methods to solve the Eikonal equation for finding the travel times of the waves. Our method is superior to the linkage-based region growing techniques since it prevents leakage and imposes compactness on the region without over-smoothing its boundary. Furthermore, we can deal with sharp corners and changes in topology. The automatic segmentation method is Eulerian, thus it is computationally efficient. We compare our results with a non-Eulerian approach that evaluates the arrival times of multiple waves as well. Our experiments illustrate the robustness, accuracy, and effectiveness of the proposed method.
\end{abstract}

Keywords: Level-set methods, image segmentation, wave propagation, Eikonal

\section{INTRODUCTION}

The level set formulation provides a general framework for modeling surface propagation. After it was introduced by the early work of Sethian, ${ }^{12}$ a large and rapidly growing body of computational techniques $\left({ }^{3}{ }_{-}{ }^{12}\right)$ from fluid mechanics to computer vision were developed to track moving interfaces in a host of setting. This framework offers a shift in how one defines moving boundaries. Typical approaches represent the motion of a curve by a discrete parameterization of the object by a set of points whose positions are updated according to a given evolution equations as in snakes, string methods, and marker particles. However, these Lagrangian techniques rely on a continual reparameterization of the surface as it becomes more complex, and they tend to disintegrate as the topology of the evolving shape changes. On the other hand, the level set method views the surface propagation as an Eulerian, initial value partial differential equation. The moving front is regarded as the zero level set of higher dimensional function. The evaluation of this higher dimensional function resembles a Hamilton-Jacobi equation, which allows forming of sharp surface gradients and incorporation of curvature effects into the propagation.

Here, we take advantage of the level set method to automatically partition an image into segments according to a consistency criterion. Previous attempts at image segmentation either suffer from requiring a priori information to initialize regions, being computationally complex, or fail to establish the color consistency and spatial connectivity at the same time. There are a wide variety of segmentation techniques that have been used for image segmentation, their algorithms are generally edge oriented, region oriented, and clustering oriented, which are either based on the concepts of similarity or discontinuity of feature values at the pixel level. Region linkage oriented segmentation grows color homogeneous regions. It starts from an initial point and extends the region by one point at a time. An adjacent pixel is added to region if the color distance between the pixel and the region is less than a threshold. This linkage process usually has no control over the shape of the current boundary. Histogram oriented techniques attempt to remedy a number of deficiencies of simple thresholding. Additionally, histogram segmentation compensates for shifts in mean intensity level since the image intensity histogram distribution is considered, rather than examining the intensity values directly, as in thresholding. However, histogram segmentation has no explicit notion of connectivity, the implicit assumption being that pixels with

\footnotetext{
*fatih@merl.com, phone: 1.617.621.7586
} 
similar intensities belong to the same regions, while this may not true in general. Edge-based segmentation takes into account that edges are boundaries between segments. After detecting edges based on searching local discontinuities, tracing algorithms are applied to link edges into continuous and connected segment boundaries. Edge linking techniques suffer from a number of problems, since not only must edge pixels be linked into edge lists, these lists must also be linked so as to extract closed region boundaries. The closure problem is not straightforward to solve, since many edges may terminate in the same location or large gaps may need to be bridged between edges. Detected edges often may not form a set of closed curves which surround connected regions.

To address the disadvantages of the above techniques, we pose the segmentation problem as monotonic wave propagation in an absorbent medium with varying front speeds. The underlying model for this method is to find the set $S$ of all similar points in image $I$ that can be connected continuously to a given initial front $\psi$ of base points. The similarity condition is determined by the color properties of the current set of points and the local color variance.

In the following section, we present the formulation of the level set method and explain the fast marching solution for the Eikonal equation, which is a simplified version thereof. In section 3, we introduce the front propagation based image segmentation method. The final section demonstrates the simulation results.

\section{LEVEL SETS AND FAST MARCHING}

The level set method combines the numerical solution of hyperbolic conversation laws into the Hamilton-Jacobi setting. Given a moving closed hypersurface $\psi(\mathbf{x}, t)$ in $R^{N}$, which is a wavefront in the segmentation process, we wish to produce Eulerian formulation for the motion of the hypersurface propagating along its normal direction with speed $F$. The propagation speed can be a function of various arguments such as a curvature, normal direction, position, etc. The main idea is to embed this propagating interface as the zero level set of a $N+1$ dimensional function $\phi$. Let the $\phi(\mathbf{x}, 0)$ is defined by $\phi(\mathbf{x}, 0)= \pm k$ where $k$ is the distance from the point $\mathbf{x}:(x, y)$ to the surface $\psi(\mathbf{x}, 0)$, Thus, we have an initial function $\phi(\mathbf{x}, 0): R^{N+1} \rightarrow R$ with the property that $\psi(\mathbf{x}, 0)=[x \mid \phi(x, 0)=0]$, i.e. the hypersurface corresponds to points that are $k=0$ away. This means the zero level set of the evolving function $\phi$ always match the propagating hypersurface $\psi$, i.e. $\phi(\mathbf{x}, t))=0$. By chain rule, we get

$$
\phi_{t}+\nabla \phi(\mathbf{x}, t) \mathbf{x}^{\prime}=0 .
$$

Since the evolution of the front on its outward normal direction is equal to the speed of the front $F=\mathbf{x}^{\prime} \cdot \nabla \phi /|\nabla \phi|$, it can easily be shown that the position of the front is becomes the zero level set of $\phi$ at time $t$

$$
\phi_{t}+F|\nabla \phi(\mathbf{x}, t)|=0
$$

If the speed function $F$ does not depend on time and preserves its sign, then the level set function $\phi(\mathbf{x}, t)=0$ becomes single-valued in $t$, i.e. each pixel is visited only once. This leads to the well-known Eikonal equation

$$
|\nabla \psi(\mathbf{x})|=F
$$

where the hyperfunction $\psi$ now corresponds to the arrival time of the front to the point $\mathbf{x}$. One of the subtleties of this equation is that the solution may be non-differantiable.

In $R^{2}$, a finite difference approximation of the gradient term in the Eikonal equation (3) can be obtained as

$$
\begin{aligned}
|\nabla \psi(\mathbf{x})|^{2} \approx & \max \left(D_{x}^{+} \psi, 0\right)^{2}+\min \left(D_{x}^{-} \psi, 0\right)^{2}+ \\
& \max \left(D_{y}^{+} \psi, 0\right)^{2}+\min \left(D_{y}^{-} \psi, 0\right)^{2} \\
= & F^{2}
\end{aligned}
$$

where the difference is chosen in terms of the direction of the flow information as

$$
D_{x}^{+} \psi=\frac{\psi(x)-\psi(x-1)}{\triangle x}, D_{x}^{-} \psi=\frac{\psi(x+1)-\psi(x)}{\triangle x}
$$

Further simplification is done by using a uniform grid, i.e. $\triangle x=\triangle y=1$. The above formulation is also called as upwind scheme, ${ }^{3}$ and it is straighforward to prove that this numerical scheme converges to the correct viscosity solution. The upwind scheme means that information propagates one way from smaller values of $\psi$ to larger values. 

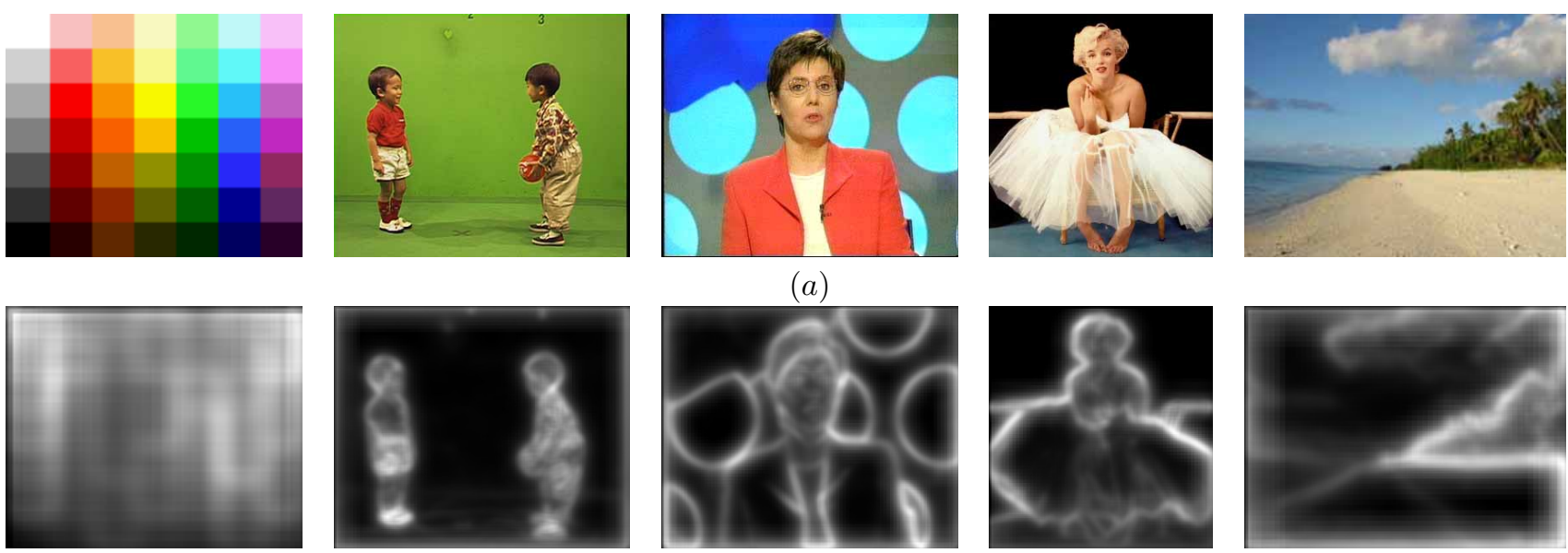

$(a)$
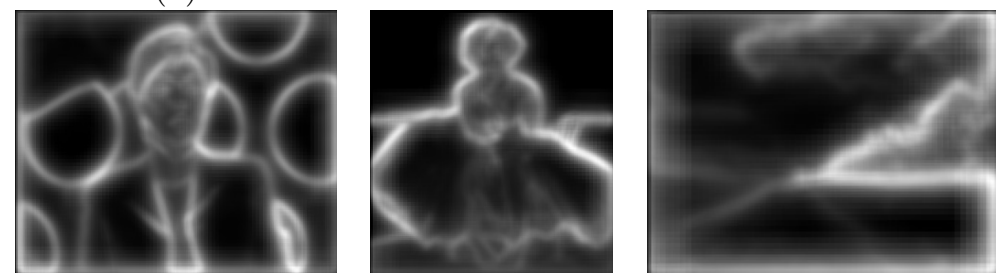

(b)

Figure 1. (a) Input images, and (b)color variance $\sigma^{2}$.

The fast marching method solves the Eikonal equation, which applies in the case of a convex, non-negative speed function. Since the front crosses each grid point only once, we can convert the moving front problem into a stationary formulation in which the arrival surface contains the information about what is moving. This can be expressed by Huygens Principle: "Every point on a primary wave front serves as the source of spherical secondary wavelets such that the primary wave front at some later time is the envelope of these wavelets". To give an example, let us consider a brick wall is being built. One stands on the current level of the wall, puts a brick, and then moves at the same level and puts another brick. Once all the bricks are placed at a given level, one then climbs up to the next level and repeats the process. The important thing is that the the wall is built from the ground up, each level must be completed before the next is begun. This is, in fact, the main difference between the linkage-based region growing and the level set method. The linkage-based region growing does not check whether a level is completed before jumping to the next level. It keeps adding bricks on the wall if there is an empty space around a brick which may easily cause the wall to extend uncontrollably and crash. The fast marching method imitates the wall building process. Starting with an initial position for the front, the method systematically marches the front outwards one grid point at a time, relying on entropy-satisfying schemes to produce the correct viscosity solution.

Similar to the brick-laying process, an Eulerian algorithm computes the arrival times of the wavefront starting from an initial front $\psi_{0}$. Let the set alive be a set of all image points $\left\{\mathbf{x}_{i}\right\}$ such that the arrival time $\psi\left(\mathbf{x}_{i}\right)=0$ is zero. Initially, this set corresponds to $\left\{\mathbf{x}_{i}: \psi\left(\mathbf{x}_{i}\right)=0\right\}$. We define a narrow band as a set of points $\left\{\mathbf{x}_{j}\right\}$ among the neighbors of the current alive set points. We assign their arrival times as $\psi\left(\mathbf{x}_{j}\right)=1 / F\left(\mathbf{x}_{j}\right)$. The rest of the points in the image are tagged as far away points. Then, we march the front forwards using the following iterative algorithm:

1. Let $\mathbf{x}^{*}$ be the point in the narrow band with the smallest arrival time $\psi\left(\mathbf{x}^{*}\right)$

2. Move $\mathbf{x}^{*}$ from the narrow band to the set of alive points

3. Move all neighbors $\mathbf{x}_{j}^{*}$ of $\mathbf{x}^{*}$ from the far away to the narrow band if they are not in the narrow band yet

4. Recompute $\psi\left(\mathbf{x}_{j}^{*}\right)$ for all neighbors of $\mathbf{x}^{*}$, i.e. solve the Eikonal equation using the quadratic approximation given in the equation (4)

5. Repeat until no point remains in the far away set.

A fast heap-sort technique can be exploited to systematically locate the proper grid point to update, so it is not necessary to backtrack over previously evaluated grid points. The resulting technique sweeps through a grid of $N$ total points in $N \log N$ steps. In the next section, we explain how the level set method is adapted for automatic segmentation of a color image. 


\section{SEGMENTATION}

We use the above wave propagation method to find the color homogeneous regions in the image. Basically, we emit wavefronts from the selected base points, and then evaluate the properties of these waves to determine the segment boundaries. In other words, a base point serves as a seed of the segment to which it belongs, and the wave propagation method functions as a tool of expansion for the segment. The segmentation algorithm is summarized as

1. Select a base point, initialize a wavefront and a segment (section 3.1)

2. Keep moving the wavefront (section 2) using the speed function (section 3.2) until

(a) Eulerian method: a stopping criteria is fulfilled (section 3.3), go to 3

(b) non-Eulerian method: all points covered, go to 4

3. Mark the current segment, and remove it from set of available points. If no point remains then stop, otherwise continue to 1 .

4. Find the maximum of the point-wise minimum arrival times. If the maximum is smaller than a threshold then stop, otherwise continue to 1 .

\subsection{Base Selection}

We iteratively select base points. A point is assigned as a base point $\mathbf{b}$ if it represents the color distribution within its local neighborhood as relevant as possible. The local color variance $\sigma^{2}$ and color gradient $\nabla I$ are indicators of the color homogeneity. The likelihood of being a base point is inversely proportional to the gradient and variance. A point has higher likelihood of being a base point if it has smaller (with respect to a global maximum) gradient and variance values, which shows the color of the point is consistent with its neighbors and color distribution is uniform around the point. This prevents from starting a wave on an edge which will disturb the color homogeneity of the segment, and enable us to effectively center the initial wavefront for more accurate segment boundary locatization. Ideally, a base point should be at the center of the segment that it belongs.

Let $I(\mathbf{x}, c)$ be the color value of the point in the corresponding color channel $c$. Assuming the color space is orthogonal, e.g. $R G B$, we define the local color variance within a local window $W$ around point as

$$
\sigma^{2}(\mathbf{x})=\sum_{c} \sum_{\mathbf{x} \in W} \omega[I(\mathbf{x}, c)-\mu(\mathbf{x}, c)]^{2}
$$

where $\mu(\mathbf{x}, c)$ is the color mean within the window and $\omega$ is an envelope function which has a Gaussian form. For the results presented in this paper, we assumed the contribution of all points within the window is same, i.e. $\omega=1$. The color gradient is given as

$$
\begin{array}{r}
|\nabla I(\mathbf{x})|=\sum_{c}|I(x+1, y, c)-I(x-1, y, c)|+ \\
|I(x, y+1, c)-I(x, y-1, c)| .
\end{array}
$$

Using the gradient and variance, we compute a dissimilarity score $d(\mathbf{x})$ for the image points

$$
d(\mathbf{x})=\alpha_{1}\left(\sum_{k}^{K-1} \psi_{k}(\mathbf{x})\right)^{-1}+\alpha_{2}|\nabla I(\mathbf{x})|+\alpha_{3} \sigma^{2}(\mathbf{x})
$$

where $K-1$ is the number of the previously emitted wavefronts, $\psi_{k}$ is the arrival time of the $k^{t h}$ wavefront, and $\alpha_{1}, \alpha_{2}, \alpha_{3}$ are constant weights. At the beginning, there is only one wavefront $K=1$, and the arrival times $\psi_{1}(\mathbf{x})$ are set to a maximum number. For the Eularian method, the contribution of the previous arrival times is dismissed, i.e. its weight is set to zero $\alpha_{1}=0$, and only the points that are not included in the previous segments are considered. For the non-Eularian 
method, all image points are evaluated. The point which has the minimum dissimilarity score is assigned as the next base point

$$
\mathbf{b}_{K}: \underset{\mathbf{x}}{\arg \max } d(\mathbf{x})
$$

Figure 1 shows sample images and their local color variances. To compute the variance within a bigger window without increasing the computational load, we applied equation 6 using the same window size( 5 times5) in a hierarchical manner at different image resolutions (original resolution, downsampled by 2 , and downsampled by 4 ). We calculated mean and variance at each level, then aggregated by compensating for the downsizing.

For each base point, we initialize a circular wavefront $\psi_{K}$ centered on the base point. Initially only the base point $\mathbf{b}_{K}$ and its 4-neighbors are on the wavefront $\psi_{K}$. The points on the current wavefront also correspond to the narrow band $B_{K}$ defined in the previous section. A segment mean color $S_{K}$ is assigned for the current segment $K ; S_{K}=I\left(\mathbf{b}_{K}\right)$.

\subsection{Wave Speed}

The ordinary Eikonal equation assumes the speed of the wave on the normal direction to the wavefront is constant. We modify the speed as a function of the color to make the propagation adaptive to the underlying color distribution. For this, three different sources of information are embedded into the speed function, namely the color similarity of the current point to the current segment, the color gradient, and the color variance. In case the color value of the current point in the narrow band is similar to the segment's color mean, the speed is increased to include the point in the segment. If a point has significantly different color value, the wavefront slows down its propagation at this point. The speed function is set to a function of color distance

$$
F(\mathbf{x})=g\left(\left\|I(\mathbf{x}), S_{K}\right\|,|\nabla I(\mathbf{x})|, \sigma^{2}(\mathbf{x})\right)
$$

where $\|\cdot\|$ is a distance norm e.g. magnitude of the difference $\left(L_{1}\right)$. A simple speed function is given by $F(\mathbf{x})=$ $\left|I(\mathbf{x})-S_{K}\right|$. At each iteration of the fast marching we update the segment color mean $S_{K}$ and the color of the narrow band $B_{K}$, the number of points $N_{K}$ in the set of alive points, and the number of points $M_{K}$ in the current narrow band as

$$
\begin{aligned}
S_{K}^{t} & =\frac{1}{N_{K}^{t}}\left[N_{K}^{t-1} S_{K}+I\left(\mathbf{x}^{*}\right)\right] \\
B_{K}^{t} & =\frac{1}{M_{K}^{t}}\left[M_{K}^{t-1} B_{K}-I\left(\mathbf{x}^{*}\right)+\sum_{j}^{C} I\left(\mathbf{x}_{j}^{*}\right)\right], \\
N_{K}^{t} & =N_{K}^{t-1}+1 \\
M_{K}^{t} & =M_{K}^{t-1}-1+C .
\end{aligned}
$$

We compute the speed of the wave using the above color mean parameters to achieve color homogeneity of the segment.

\subsection{Stop Criteria}

An important problem of region growing is the determination of the stopping conditions. We propose four different criteria for the Eulerian approach.

One possible measure the color similarity of the segment and the current point.

$$
\left\|S_{K}-I\left(\mathbf{x}^{*}\right)\right\|<\tau_{A}
$$

that is if the color value of the current point is more that the threshold $\tau_{A}$ then the wavefront frozen at that point. This threshold may be set to a constant. We observed that a threshold $\tau_{A}=3 \cdot 2^{m} / 64$ (assuming color channels are $m$-bit coded) produces accurate segmentation for most test images. We obtained this value empirically.

Another measure does not only make comparison between the point and the segment but compares the whole wavefront with the segment.

$$
\left\|S_{K}-B_{K}\right\|<\tau_{B}
$$

Here, the threshold is similar to the previous color threshold $\tau_{B} \approx \tau_{A}$. 

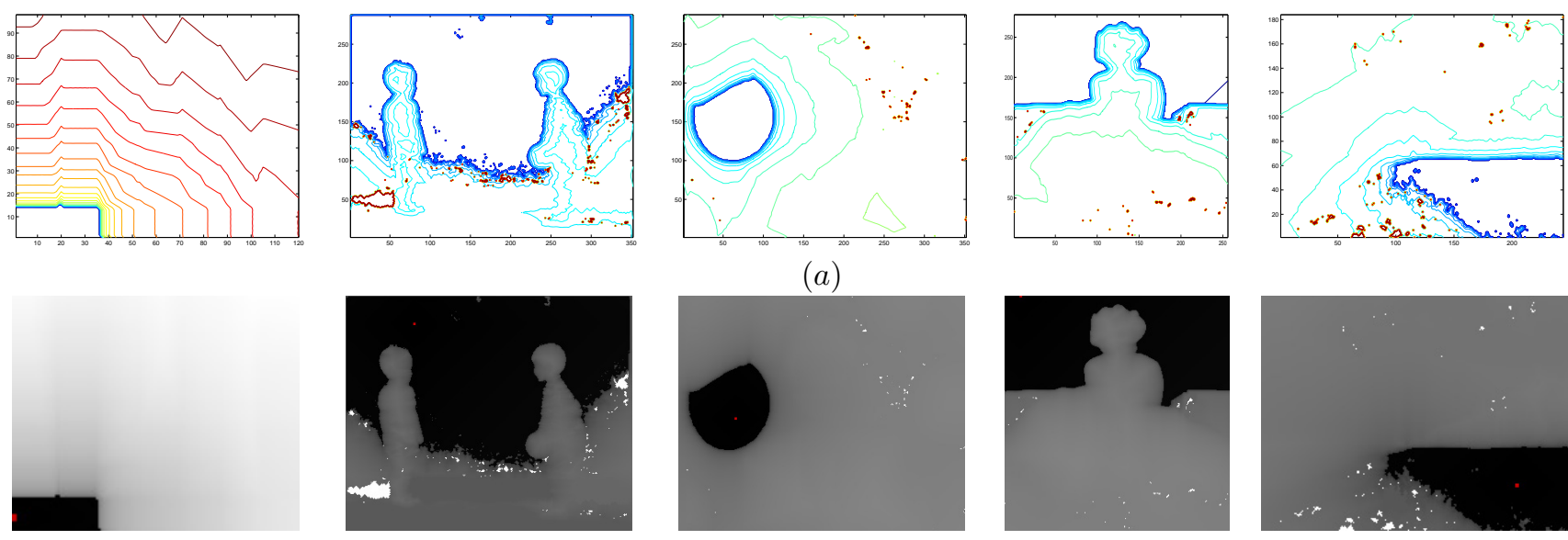

(a)
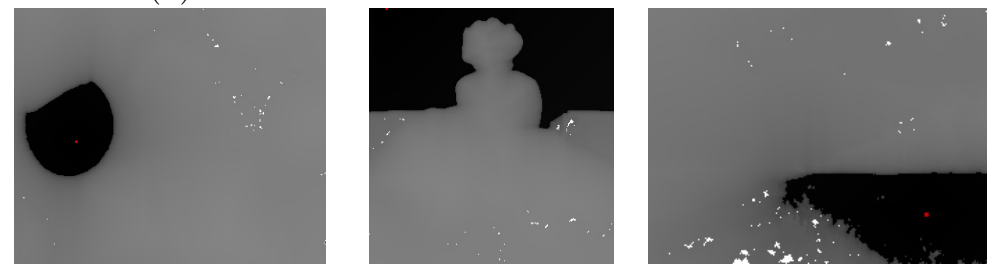

(b)

Figure 2. (a) Propagation of the first wavefronts. (b) Arrival times $\psi_{1}(\mathbf{x})$ and the first selected base points $\mathbf{b}_{1}$ (marked in red).

Yet, we can expand the segment up to the edges:

$$
\left|\nabla I\left(\mathbf{x}^{*}\right)\right|<\tau_{C} .
$$

However this criterion relies on the assumption that the speed is constant thus the wavefront can continue moving until it reaches an edge. In this formulation, a point that has larger gradient values than the threshold $\tau_{C}$ is considered as an edge point. In case the wavefront reaches an edge, it stops marching at that point and the propagation continues until no more expansion is possible.

Alternatively, for a non-constant wavefront speed function, the arrival time $\psi(\mathbf{x})$ of the wavefront can be employed as a stopping criteria. The wave continues its propagation if

$$
\psi\left(\mathbf{x}^{*}\right)<\tau_{D}
$$

It is necessary to consider the effect of the image size and the parameterization of the speed function when we assign the threshold. If the threshold $\tau_{D}$ is not scaled with the image dimensions, a wavefront may stop prematurely in case of a large segment.

\section{DISCUSSION}

We tested the proposes method using a non-constant speed function

$$
F(\mathbf{x})=\alpha_{1}\left|S_{K}-I(\mathbf{x})\right|+\alpha_{2}|\nabla I(\mathbf{x})|+\alpha_{3}
$$

where $\alpha_{1}>\alpha_{2}>\alpha_{3}$. The constant term $\alpha_{3}$ incorporates the effect of the distance from the base point, thus it serves as a reinforcement on the shape of the wavefront. In other words, the constant term acts as a viscosity parameter and controls the curvature of the front. Note that, in case of $\alpha_{3} \gg \alpha_{1}$ the wavefront moves forward at a constant speed by disregarding the color similarity of the front to segment.

Fig. 2-a shows the motion of the wavefronts for the first base points $\mathbf{b}_{1}$. The arrival times $\psi_{1}$ are shown in Fig.2-b where the base points are marked as red, and higher intensity values indicates larger the arrival times. As visible from the graphs, the wavefront can successfully modify its shape to match with the underlying color distribution. The extracted segments are given in Fig. 3. The first row shows the color coded segments of the Eulerian approach. As the stop criterion, we compared the wavefront arrival times (eq. 15). The threshold is kept same for all the test images. It is observed that the boundary locatization accuracy is not sensitive to the selection of the threshold which shows robustness of the proposed method.

The color space choice also is an important component of the segmentation problem. To illustrate this, we applied the same method using the normalized $r, g, b$ color space $(r=R /(R+G+B), \ldots)$. The results are given in Fig.3-b. 

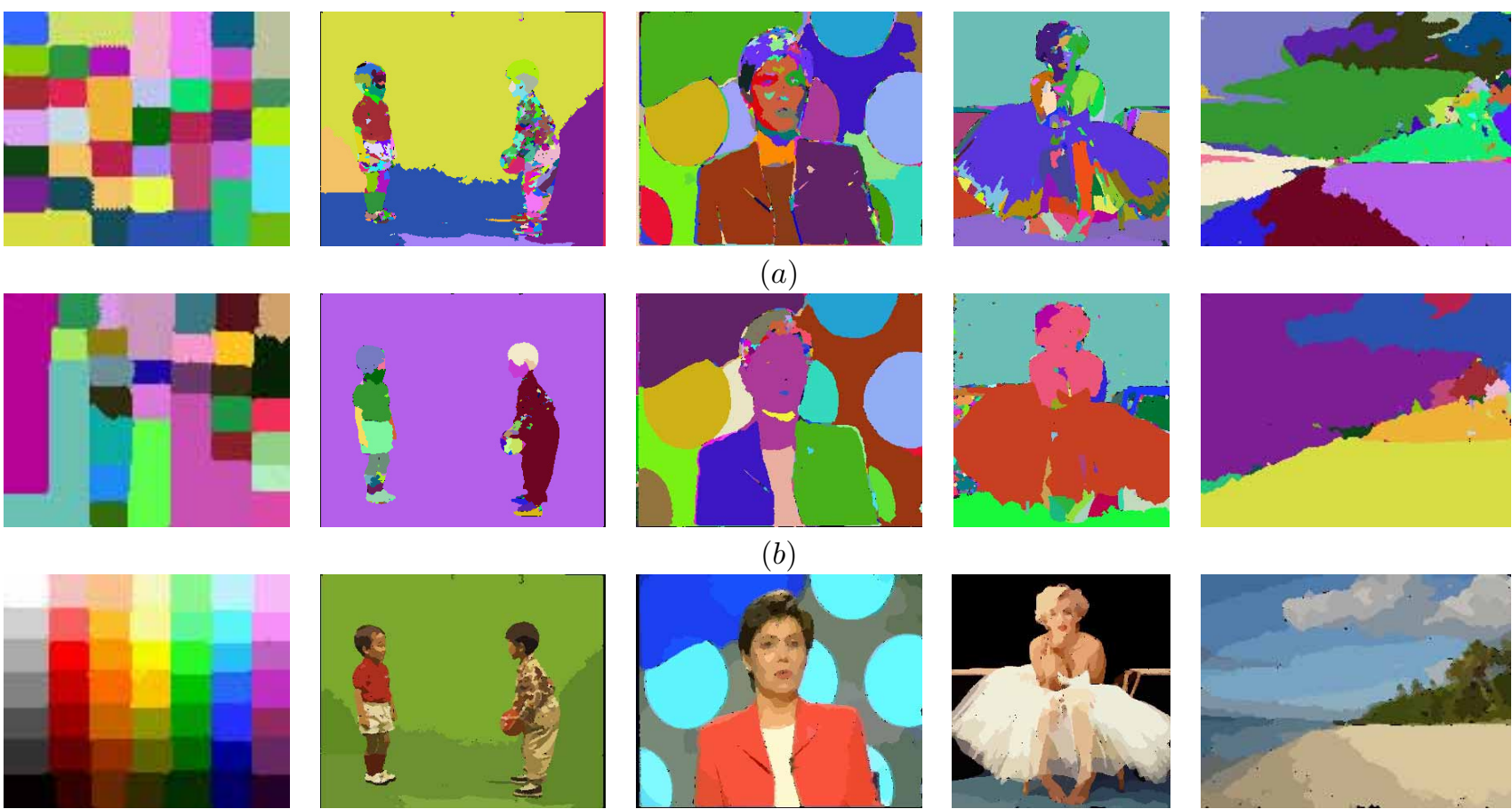

(a)
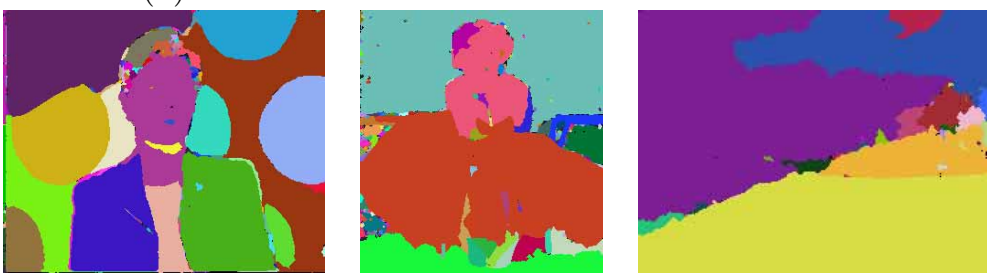

$(b)$
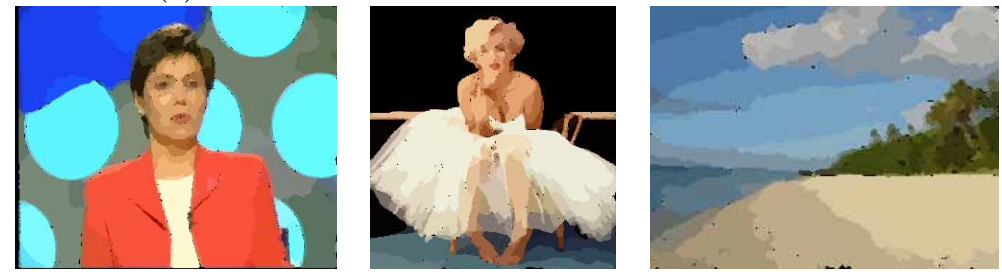

$(c)$

Figure 3. (a) Segmentation results ( $R G B$ color space). Each segment is coded by a random color. (b) Segmentation results ( $r g b$ color space). (c) Final mean segment colors $S_{K}(R G B$ color space).

\section{SUMMARY}

In this paper, we propose a wavefront propagation based image segmentation method to overcome the limitations of the existing region growing techniques. We formulate a color similarity and gradient based speed function, four different propagation stop criteria.

Using the level set techniques, we achieve integration of the spatial continuity, boundary shape control mechanism, and color consistency at the same time in an adaptive framework. This is an important improvement of the ordinary linkagebased region growing approach. Furthermore, the fast marching solution of the Eikonal equation effectively reduces the computational cost of the segmentation process. Our experiments illustrate the robustness, accuracy, and effectiveness of the proposed method.

We are currently investigating the relationship of the speed function parameters and the stop criteria thresholds. We will include a detailed analysis of the four stop criteria and an automatic threshold adaptation method in the final version of this paper. We will also include the comparison of the described non-Eulerian approach and a table of the computation time performances.

\section{REFERENCES}

1. J. A. Sethian, "An analysis of flame propagation", Ph.D. Dissertation, Mathematics, University of Berkeley, 1982

2. J. A. Sethian, "Curvature and evaluation of fronts", Commun. in Mathematical Physics, Vol. 101, pp. 487-499, 1985

3. S. Osher and J. A. Sethian, "Fronts propagating with curvature dependent speed: Algorithms based on Hamilton-Jacobi formulation”, Journal of Computational Physics, Vol. 79, pp. 12-49, 1988

4. G. Koepfler, C. Lopez, and J. Morel, "A multiscale algorithm for image segmentation by variational method", SIAM J. of Num. Analysis, 31-1, pp. 282-299, 1994

5. R. Malladi, J. A. Sethian, and B. Vemuri, "Shape modelling with front propagation: a level set approach", IEEE Trans. on Pattern Analysis and Machine Intelligence, Vol. 17, pp. 158-175, 1995 
6. O. Faugeras and R. Keriven, "Variational Principles, Surface Evolution, PDE's, level set methods and the Stereo Problem", IEEE Transactions on Image Processing, Vol. 7, No. 3, pp 336-344, 1998

7. K. Siddiqi, A. Tannenbaum, and S. W. Zucker. "A Hamiltonian Approach to the Eikonal Equation”. Workshop on Energy Minimization Methods in Computer Vision and Pattern Recognition, 1999

8. M. Droske, T. Preuer, and M. Rumpf, "A multilevel segmentation method”, Proc. Vision, Modeling and Visualization, MPI Informatik, Germany, pp. 327-336, 2000

9. M. Leventon, Olivier Faugeras, Eric Grimson, William Wells, "Level Set Based Segmentation with Intensity and Curvature Priors", IEEE Workshop on Mathematical Methods in Biomedical Image Analysis, 2000

10. B.C. Vemuri, J. Ye, Y. Chen, C.M. Leonard, "A Level-Set Based Approach to Image Registration", IEEE Workshop on Mathematical Methods in Biomedical Image Analysis, 2000

11. N. Paragios and R. Deriche, "Geodesic active regions and level set methods for supervised texture segmentation" International Journal of Computer Vision Vol.46, pp 223, 2002

12. T.F. Chan, J. Shen and L. Vese, "Variational PDE Models in Image Processing", Notices of the American Mathematical Society, 2003 